\title{
Heat transfer enhancement of a periodic array of isothermal pipes
}

\author{
Theodoros Leontiou*, Magzhan Ikram ${ }^{\dagger}$, \\ Kenes Beketayev $\ddagger$ and Marios M. Fyrillas ${ }^{\S *}$ \\ *General Department, Frederick University, 1303 Nicosia, Cyprus. \\ ${ }^{\dagger}$ National Laboratory Astana. \\ ${ }^{\ddagger}$ Computer Science Department, \\ $\& \S$ Department of Mechanical Engineering, \\ Nazarbayev University, Astana 010000, Republic of Kazakhstan.
}

February 18, 2016

\begin{abstract}
We address the problem of two-dimensional heat conduction in a solid slab whose upper and lower surfaces are subjected to uniform convection. In the midsection of the slab there is a periodic array of isothermal pipes of general cross section. The main objective of this work is to find the optimum shapes of the pipes that maximize the Shape Factor (heat transport rate). The Shape Factor is obtained by transforming the periodic array of pipes into a periodic array of strips, using the generalized SchwarzChristoffel transformation, and applying the collocation boundary element method on the transformed domain. Subsequently we pose the inverse problem, i.e. finding the shape that maximizes the Shape factor given the perimeter of the pipes. For large Biot number the optimum shapes are in agreement with the isothermal case, i.e. circular for sufficiently small perimeters/heat transfer, and elongated towards the surfaces of the slab for larger perimeters/heat transfer. Furthermore, for the isothermal case, we were able to discover a new family of optimum shapes for large thickness of the slab and large
\end{abstract}

\footnotetext{
*E-mail: t.leontiou@frederick.ac.cy

${ }^{\dagger}$ E-mail: magzhan.ikram@nu.edu.kz

${ }^{\ddagger}$ E-mail: kenes.beketayev@nu.edu.kz

${ }^{\S}$ Corresponding Author, e-mail: m.fyrillas@gmail.com
} 
perimeters, which do not have their maximum width on the horizontal axis of symmetry. For small Biot number the optimum pipes are flatter than the isothermal ones for a given perimeter. The flatness becomes more apparent for larger perimeters. Most important, for large perimeters there exists a critical thickness which is characterized by maximum heat transfer rate. This is further investigated using the finite element method to obtain the critical thickness of a slab and the critical depth of the periodic array of circular pipes.

\section{Keywords}

Solid slab with periodic array of pipes; Convective heat transfer; Laplace equation, generalized Schwarz-Christoffel transformation; Shape Optimization, Optimum shapes of pipes; Critical Thickness of slab, Critical depth of the pipes.

\section{Introduction}

In this work we address the problem of heat conduction in a solid slab embedded with a periodic array of isothermal pipes; the surfaces of the slab are subjected to convection with a uniform/constant convection heat transfer coefficient [1]. The shape of the pipes is assumed unknown and the main objective of this work is to find the shape that maximizes heat transfer. The particular configuration is a classical heat conduction problem that arises in connection with heating tubes, oil lines, steam distribution lines, underground electrical power-line transmission, laser sintering processes, in certain types of compact heat exchangers and solar cells [2-10].

A similar problem has been addressed by Fyrillas [11] where, however, the surfaces of the slab were assumed to be isothermal. When the slab is subjected to uniform convection, Fyrillas \& Stone [12] showed that there exists a critical insulation thickness associated with a slab embedded with a periodic array of isothermal strips. Similarly, Fyrillas \& Leontiou $[13,14]$ also showed that there is a critical thickness associated with a fin that is subjected to uniform convection.

Following the analysis in $[11,15-19]$, the physical domain is transformed into a rectangular channel using the generalized Schwarz-Christoffel transformation [20-23]. The heat 


\begin{tabular}{|ll|}
\hline Nomenclature & \\
$B i$ & Biot number $=L h / k$ \\
$h$ & Green's function \\
$h$ & one half the thickness of the slab in the complex domain \\
$H$ & convection heat transfer coefficient $\mathrm{W} /\left(\mathrm{m}^{2} \mathrm{~K}\right)$ \\
$k$ & one half the thickness of the slab in the physical domain (dimensionless) \\
$L$ & thermal conductivity W/(m $\mathrm{K})$ \\
$P$ & dimensional distance between two consecutive pipes (period, $\mathrm{m})$. \\
$S$ & Length-scale used for non-dimensionalization \\
$T$ & one half the perimeter of the pipe (dimensionless) \\
$x, y$ & Shape Factor (dimensionless) \\
$z$ & temperature, $\mathrm{K}$ \\
$z_{i}$ & coordinates of the physical plane (dimensionless) \\
Symbols & complex coordinate of the physical plane \\
$\alpha_{i}$ & vertices in the physical plane \\
$\xi, \eta$ & \\
$w=\xi+$ i $\eta$ & turning angles divided by $\pi$ \\
$w_{i}$ & coordinates of the transformed domain \\
Subscripts & image of $z_{i}$ vertices in the transformed domain \\
$i$ & \\
Diacritic & related to the $i$-th vertex \\
$\wedge$ & \\
\end{tabular}

transfer problem in the transformed domain is addressed numerically using the "singular" boundary element method [24-30].

As mentioned earlier, the main objective of this work is to pose and solve a Shape Optimization problem, i.e. an inverse design problem, where the objective function is the Shape Factor $[1,31]$, i.e. the total heat transfer rate, and the variable of the optimization is the shape of the pipe, which is parameterized though the parameters of the generalized Schwarz-Christoffel transformation. Hence, using the parameters of the generalized Schwarz-Christoffel transformation, the Shape Optimization problem is posed as a nonlinear programming problem (constrained nonlinear optimization [32]), which is solved numerically [33] to find optimum shapes that maximize heat transfer. We should point out that 
the application of the generalized Schwarz-Christoffel transformation confines the Shape Optimization to simply-connected domains. For general domains, one needs to consider a conformal transformation for multiply-connected domains [23] that might not be available. In such domains, although the direct problem can be solved in the physical domain using the boundary element method [24] the inverse problem, i.e. the shape optimization problem, would be intractable. It can be addressed by considering a more specific geometric parametrization and use boundary element methods if the governing PDE is the Laplace equation [34-37], or finite element methods for more general problems [38-41]. Although it is tempting to infer that conformal mapping techniques provide a natural basis for Shape Optimization problems associated with the Laplace equation in simply-connected domains, for general cases one needs to consider more general formulations [42-44].

The case of a single pipe in an infinite domain was treated in [16] where it was shown that the circular shape is the optimum shape for both maximization and minimization problems. In addition it was shown that: (i) the heat transport rate maximization problem, for a given perimetric length, is equivalent (dual) to the perimeter minimization problem for a given transport rate; and that (ii) the heat transport rate minimization problem, for a given area of the cross section, is equivalent (dual) to the area maximization problem for a given transport rate.

The duality of the shape optimization problems was also shown to apply for the case of a single isothermal pipe embedded in a slab (bounded domain), where the upper and lower surfaces of the slab are maintained at a constant temperature [17], while the slab is infinite in the horizontal direction. A circular shape is the optimum shape in the limit of small transport rates, i.e. the thickness of the slab is large. For larger transport rates, the optimum shapes tend to elongate towards the surfaces of the slab for the Shape Factor maximization problem, while it is elongated in the horizontal direction for the Shape Factor minimization problem. It is interesting to note that the optimum shape of the pipe does not extend beyond the half thickness of the slab for the Shape Factor minimization problem $[45,47,46]$.

The case of a periodic array of isothermal pipes was treated in [11] and [19]. In the former work, both surfaces of the slab were assumed isothermal while in the latter, the 
lower surface was assumed adiabatic. In general the results suggest that, for the Shape Factor maximization problem, the optimum shapes are elongated towards the isothermal surfaces of the slab because this leads to a large temperature gradient due to the proximity of the pipe to the isothermal surfaces of the slab, hence a high transport rate is achieved. As far as the duality between the shape optimization problems is concerned (described in the previous paragraphs), for a periodic domain there is no rigorous prove of its existence. However, it has been justified through numerical simulations.

The existence of a critical thickness associated with a slab subjected to convection and embedded with isothermal strips [12], establishes that there is a significant difference between an isothermal slab and a slab subjected to convection. Hence, in the current work we investigate the optimum shape of the pipes when the surfaces of the slab are subjected to convection. In the next Section ( $(2)$, we describe briefly the numerical solution of the problem, i.e. the conformal mapping technique and the boundary element method. In Section $\S 3$ we pose and solve numerically the Shape Optimization problem of finding the optimum shape such that the heat transfer rate is maximized. In Section $\S 4$, using finite element simulations, we verify the existence of a critical thickness associated with a slab embedded with a periodic array of circular pipes when the slab is subjected to convection. In addition it is revealed that there exists a critical depth associated with pipes embedded in an insulated slab. We summarize our findings in the last Section.

\section{Shape factor of a periodic array of isothermal pipes}

The analysis of this section closely follows the definitions and notation outlined in [11], where the surfaces of the slab were assumed to be isothermal. In this work we assume that the surfaces of the slab are subjected to uniform convection with a uniform heat transfer coefficient $(h)$, hence the work considered in [11] is the asymptotic limit of the present analysis for $h \rightarrow \infty$ (large Biot number, $B i \rightarrow \infty$ ), i.e. strong convection.

Consider heat conduction due to a periodic array of isothermal $\left(T_{1}\right)$ symmetric pipes of general cross section, embedded at the center of a solid slab. The temperature field is governed by the Laplace equation (Fig. 1). The upper and lower surfaces of the slab are subjected to convection with a constant convection heat transfer coefficient $(h)$ and a 
constant far-field temperature $T_{\infty}[1]$. We non-dimensionalize lengths with the distance between two consecutive pipes $(L)$, i.e. the period, and the temperature by subtracting $T_{\infty}$ and dividing by the temperature difference $T_{1}-T_{\infty}$. The dimensional analysis leads to the following definition for the Biot number $B i=L h / k$, where $k$ is the thermal conductivity. In addition, because of symmetry, we consider only the upper half of the region (the formulation is also relevant for a periodic array of isothermal objects embedded in a large, non-conducting substrate), and that the left point of symmetry of one pipe coincides with the origin of the coordinate system. The domain and the dimensionless parameters associated with the problem are clearly indicated in Fig. 1.

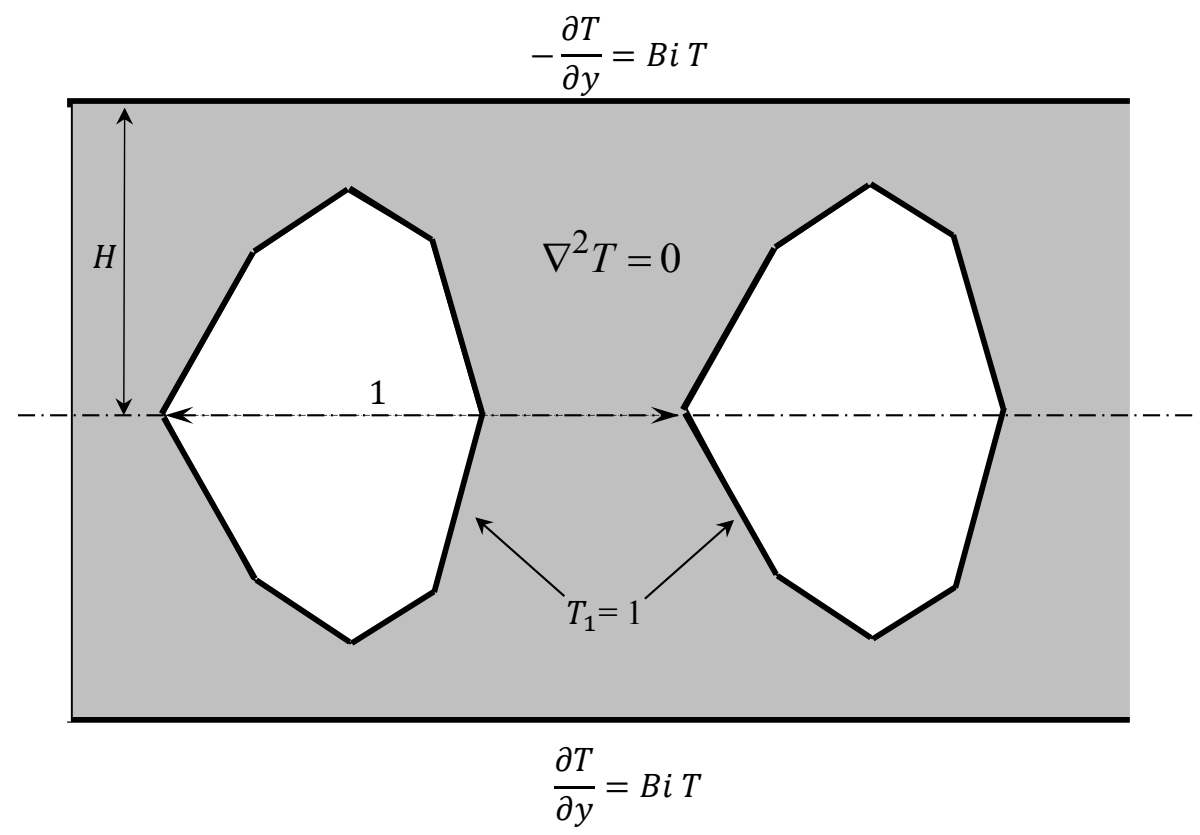

Figure 1: Schematic representation of the physical problem and the associated dimensionless parameters. The surfaces of the slab are subjected to uniform convection and the dimensionless distance between them, i.e. the period, is 1. At the mid-distance there is a two-dimensional, periodic array of isothermal symmetric pipes $T=1$, of general cross section. The dimensionless half-thickness of the slab is $H$. Length units have been nondimensionalized by the period $L$.

By applying the generalized Schwarz-Christoffel transformation (Appendix A), we map the physical domain onto a finite channel embedded with a periodic array of isothermal strips (Fig. 2). The problem associated with our analysis is that of heat conduction in a channel where the upper surface is subjected to convection with a variable convection heat transfer 

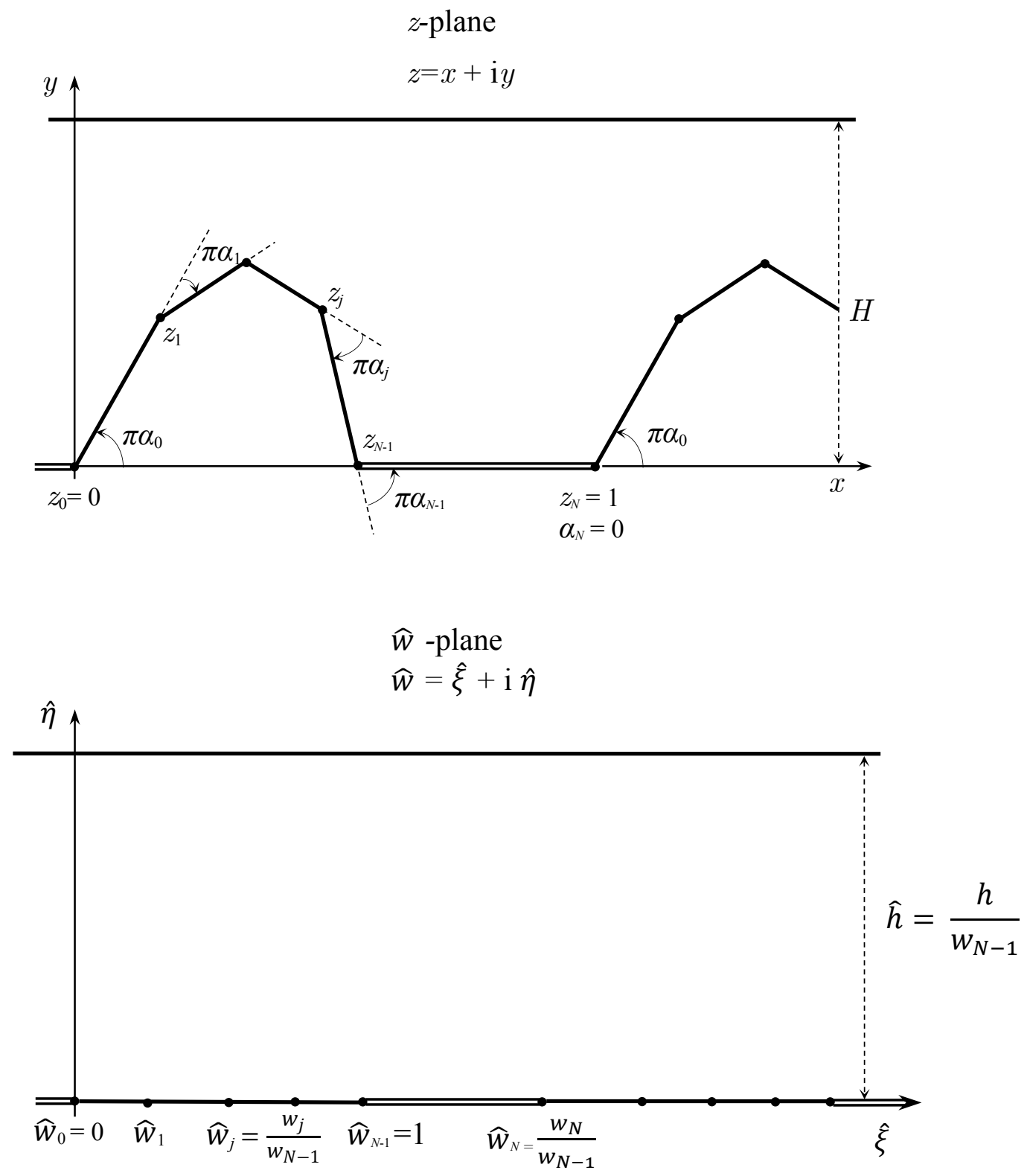

Figure 2: Mapping of the physical domain onto the upper-half plane using the generalized Schwarz-Christoffel transformation (equation 4). Lengths in the physical and complex domain are non-dimensionalized with $L$ and $w_{N-1}$, respectively.

coefficient which is equal to $B i\left|\frac{\mathrm{d} z}{\mathrm{~d} \hat{w}}\right|$. This is a consequence of the conformal coordinate transformation [26]. At the lower surface of the channel there is a two-dimensional (infinite span), periodic array of isothermal strips of unit length and period $\hat{w}_{N}$, which are kept at dimensionless temperature $T=1$. At steady-state, the temperature distribution is governed 
by the Laplace equation $\nabla^{2} T=0$. In view of periodicity in the $\hat{\xi}$ direction, the boundary conditions are as follows:

$$
\begin{aligned}
& \text { On } \hat{\eta}=0\left\{\begin{array}{lll}
T[\hat{\xi}]=1 & \text { along } & 0 \leq \hat{\xi} \leq 1 \\
\frac{\partial T}{\partial \hat{\eta}}[\hat{\xi}]=0 & \text { along } 1<\hat{\xi}<\hat{w}_{N}
\end{array}\right. \\
& \text { On } \hat{\eta}=\hat{h} \quad \frac{\partial T}{\partial \hat{\eta}}[\hat{\xi}]+B i T[\hat{\xi}]\left|\frac{\mathrm{d} z}{\mathrm{~d} \hat{w}}\right|_{\hat{w}=\hat{\xi}+\mathrm{i} \hat{h}}=0 \\
& T[\hat{\xi}=0, \hat{\eta}]=T\left[\hat{\xi}=\hat{w}_{N}, \hat{\eta}\right] .
\end{aligned}
$$

The mathematical model along with the boundary conditions are shown in Fig. 3.

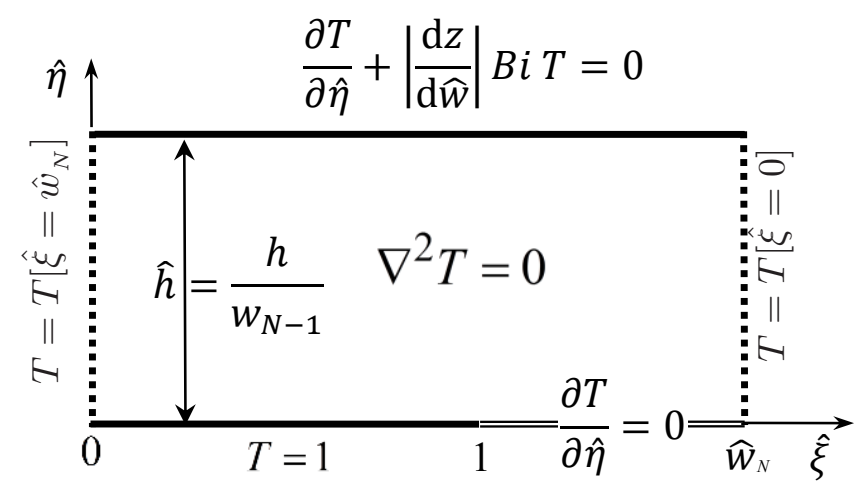

Figure 3: Schematic representation of the model problem along with boundary conditions. The Neumann boundary is dictated by the symmetry of the configuration in the $\hat{\eta}$-direction.

In Appendix B, using the boundary element method [24, 25], we obtain an integral formulation of the problem associated with a single strip which is solved numerically. The objective is to determine the heat transfer rate (transport rate) for the configuration shown in Fig. 3. Equivalently, we define the Shape Factor $(S)$ [1] associated with a single strip of unit span:

$$
S=-\int_{0}^{1} \frac{\partial T}{\partial \hat{\eta}}[\hat{\xi}, \hat{\eta}=0] \mathrm{d} \hat{\xi} .
$$

where we only consider the upper-half of the domain because of symmetry.

\section{Shape Optimization}

The formulation of the Shape Optimization problem follows along the same lines as the problems formulated in $[16,17,11,18,19]$. The objective function is the Shape Factor (Eq. 
2)

$$
\underset{\left(\boldsymbol{\alpha}, w_{N-1}, \hat{w}_{N}\right)}{\operatorname{maximize}} S\left[\boldsymbol{\alpha}, w_{N-1}, \hat{w}_{N}\right]
$$

and the constraints are dictated in view of the geometrical configuration (Fig. 2, Eq. 6):

$$
\begin{aligned}
& \sum_{j=0}^{N-2}\left|z_{j+1}-z_{j}\right|=P, \\
& \operatorname{Re}\left[z_{N}\right]=x_{N}=1, \\
& \operatorname{Im}\left[z_{N}\right]=y_{N}=0, \\
& \sum_{j=0}^{N} \alpha_{j}=0, \\
& \alpha_{N}=0 .
\end{aligned}
$$

The geometrical parameters $P$ and $H$ (Eq. 5, Fig. 1) are assigned a priori along with the Biot number $(B i)$. The variables of the optimization are the parameters of the SchwarzChristoffel transformation $\boldsymbol{\alpha}$, which are equal to the turning angles between the design elements multiplied by $\pi$, and $\left(w_{N-1}, \hat{w}_{N}\right)$ which are related to the locations of the last two vertices in the transformed domain.

In the next section we solve numerically the Shape Optimization problem (Eq. 3) for different values of the geometrical parameters $P, H$ and the Biot number $(B i)$.

\subsection{Numerical Results}

In this work, similar to $[16,17,11,18,19]$, we have used conformal mapping techniques to formulate the Shape Optimization problem of maximizing the Shape Factor (Eq. 3) as a nonlinear programming problem (constrained nonlinear optimization), i.e. find the constrained extremum of a scalar function of several variables, where the variables are the parameters of the generalized Schwarz-Christoffel transformation. The Shape Optimization problem under consideration is solved using the NLPQL code developed by Schittkowski [33], which uses a special implementation of a sequential quadratic programming (SQP) method.

As discussed in the above mentioned references, the Schwarz-Christoffel integral (Eq. 4) is evaluated using Gauss-Jacobi quadrature, as outlined in [23], and the infinite product appearing in the integral can be truncated to a small value without affecting the accuracy due to the exponential decay of the hyperbolic sines [22]. 
With the exception of $\hat{w}_{N}$, we choose the $\hat{w}_{j}$ s to be equispaced between 0 and 1 except near large slopes, i.e. when the $\alpha_{j}$ s are large, where we include extra points in order to improve the resolution of the shape. Although this refinement is limited due to crowding [23], the computations converge to a particular geometry. We should point out that the elements of the Shape Optimization procedure, i.e. the parametrization of the pipe using the generalized Schwartz-Christoffel transformation (Eq. 4, Fig. 2), are not the same with the elements used for the solution of the boundary element problem (Eqs. 9, Fig. 3). Although 10-30 elements are used for Shape Optimization (Figs 4-7), for the solution of the integral equations (Eqs. 9) and consequently the calculation of the shape factor (Eq. 2), initially 100 elements are used and are successively refined to achieve a relative accuracy of $1 \%$. The latter was computationally intensive and a parallel version of the boundary element numerical procedure was developed. As a starting vector, for the shape optimization, we have used $\boldsymbol{\alpha}=0$, however the accuracy of the converged solution was verified by repeating the calculation with smaller tolerances and a different starting vector. Although this does not provide a rigorous proof that the optimum is global, within the limitations of the parametrization using the Schwarz-Christoffel transformation, our numerical optimization procedure is consistent and stable.

In Figs. 4 and 5, we show numerical solutions of the shape optimization problem for the isothermal case, i.e. the case considered in [11], and for the case of large Biot number $(B i=1000)$, for heights $H=0.1$ and $H=2$. The results for the isothermal case coincide with the large Biot number case, and the two curves are indistinguishable, i.e. the two optimum shapes coincide and they are equivalent. Furthermore, in agreement with [11], for small heat transfer rates the optimum shapes are circular while for larger heat transfer rates the optimum shapes become successively elliptical, elongated in the vertical direction, approaching the isothermal surfaces of the slab. Further to the aforementioned classical results/shapes [11], a different family of optimum shapes have been revealed in the case of large thickness of the slab (Fig. 5) and large perimeter of the pipe; these shapes do not have their maximum width along the horizontal axis of symmetry of the pipe. For comparison, in Figs. 6 and 7 we show results of the numerical shape optimization for a small Biot number, i.e. $B i=0.1$, for heights $H=0.1$ and $H=2$ respectively. The optimal 


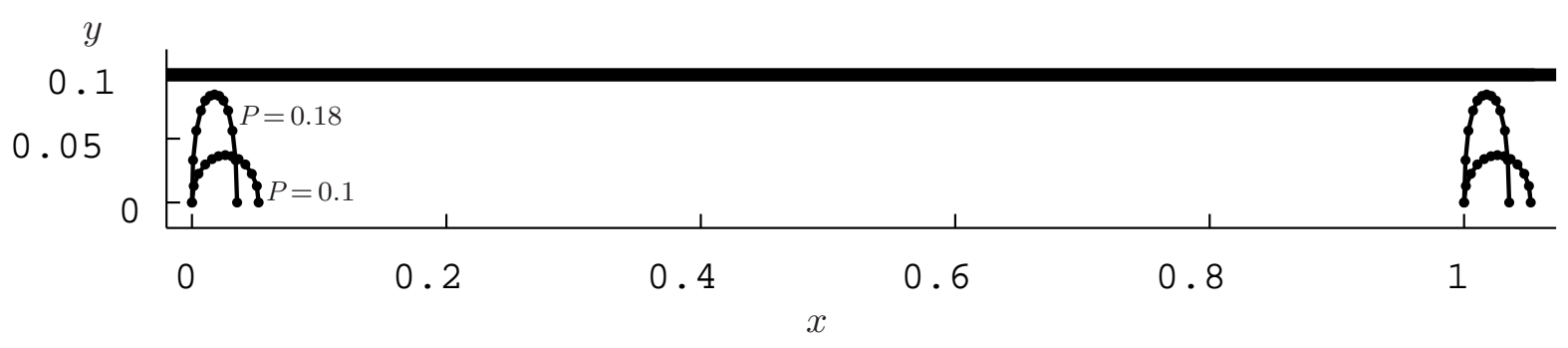

Figure 4: Optimum shapes that maximize the Shape Factor when the dimensionless height is $H=0.1$ and the surfaces of the slab are isothermal [11] or characterized by large Biot number $(B i=1000)$; the optimum shapes are indistinguishable. The two optimum geometries shown correspond to $P=0.1, S=2.275$; and $P=0.18, S=4.614$.

shapes are flatter than the corresponding large Biot number case. This characteristic is further addressed and explained in the following paragraphs.

In Fig. 8 we evaluate the accuracy of the optimization procedure and the influence of the design points. As a test case we consider one of the optimum shapes that does not have its maximum width along the horizontal axis of symmetry of the pipe, in view of the large length of the first element. In particular, we consider one of the cases of Fig. 7 and re-run it with twice the number of points. We should point out that the length of the initial element cannot be refined further due to crowding [23]. Comparing the two shapes in Fig. 8, we conclude that the shape optimization procedure is both stable and consistent, however the convergence is limited when the optimum shape is susceptible to crowding.

The optimum shapes are justified if one considers how the temperature distribution on the surface of the slab is affected by the ratio of the perimeter of the pipe with the thickness of the slab. If the ratio is relatively small, then the temperature on the surfaces of the slab is rather uniform and the optimum shape is similar to the isothermal case. On the other 


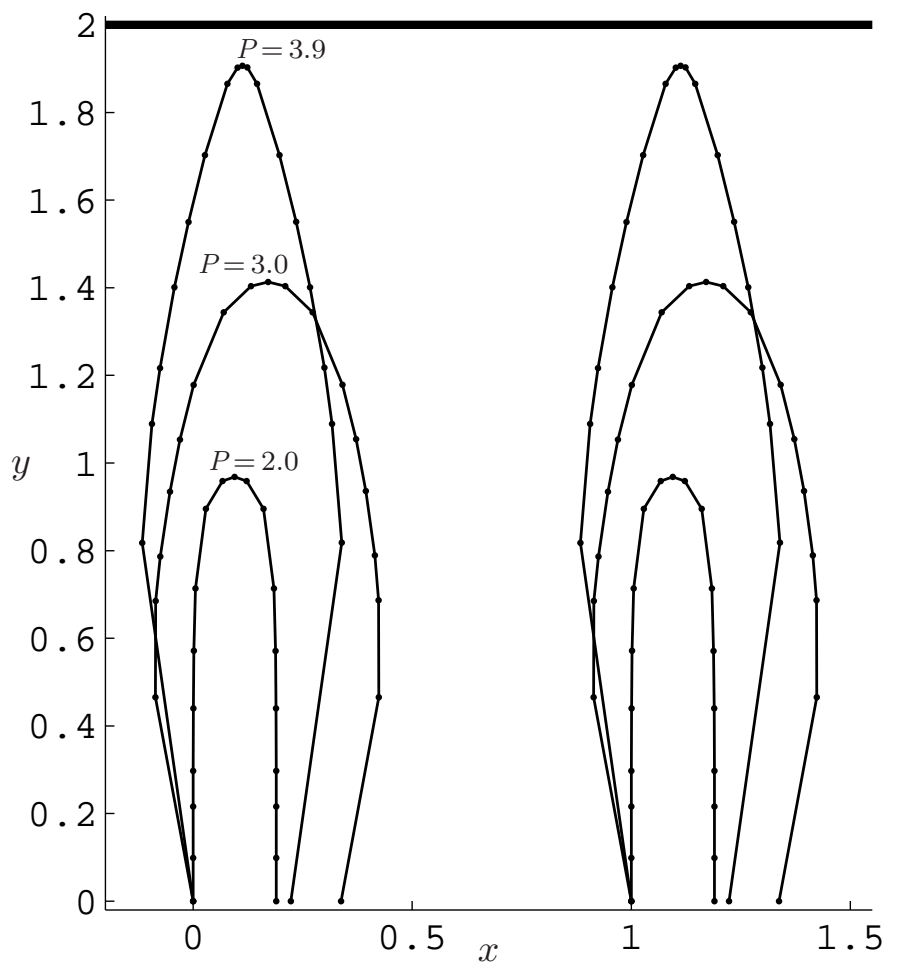

Figure 5: Optimum shapes that maximize the Shape Factor when the dimensionless height is $H=2$ and the surfaces of the slab are isothermal [11] or characterized by large Biot number $(B i=1000)$; the optimum shapes are indistinguishable. The three optimum geometries correspond to $P=2.0, S=0.836 ; P=3.0, S=1.518$; and $P=3.9, S=7.121$.

hand if the ratio is relatively large, then the temperature distribution on the surfaces of the slab is not uniform, and the optimum shapes differ from the isothermal case. Another characteristic of the optimum shapes at small Biot number is that they are flatter than the isothermal case, and actually there seems to exist a critical thickness. This is justified by considering the temperature gradient along the surface of the slab; if the shape is elongated towards the surface of the slab, the temperature gradient would be small because the surface of the slab would approach the pipe temperature. But if the temperature approaches the pipe temperature the convection would be enhanced. Hence, there is an optimum balance between conduction and convection similar to the cases considered in $[12,14,13]$, and the classical case of the critical thickness of insulation associated with circular pipes [1]. This is investigated further in the next section where we consider the heat transfer associated 
with a periodic array of isothermal circular pipes embedded in a slab whose surfaces are subjected to uniform convection.

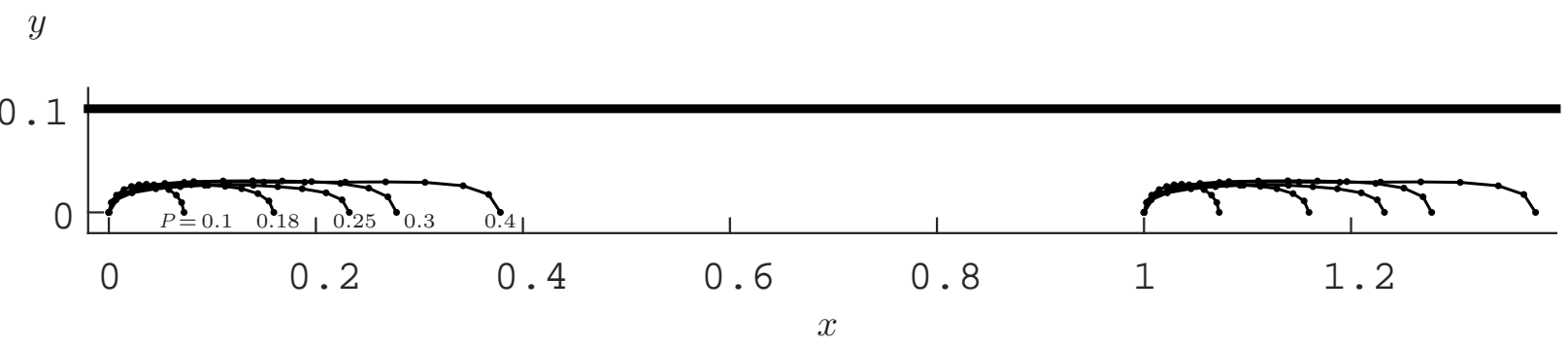

Figure 6: Optimum shapes that maximize the Shape Factor when the dimensionless height is $H=0.1$ and the $B i=0.1$. The five shapes correspond to $P=0.1, S=0.092 ; P=$ $0.18, S=0.0936 ; P=0.25, S=0.0948 ; P=0.3, S=0.096$; and $P=0.4, S=0.0967$. 


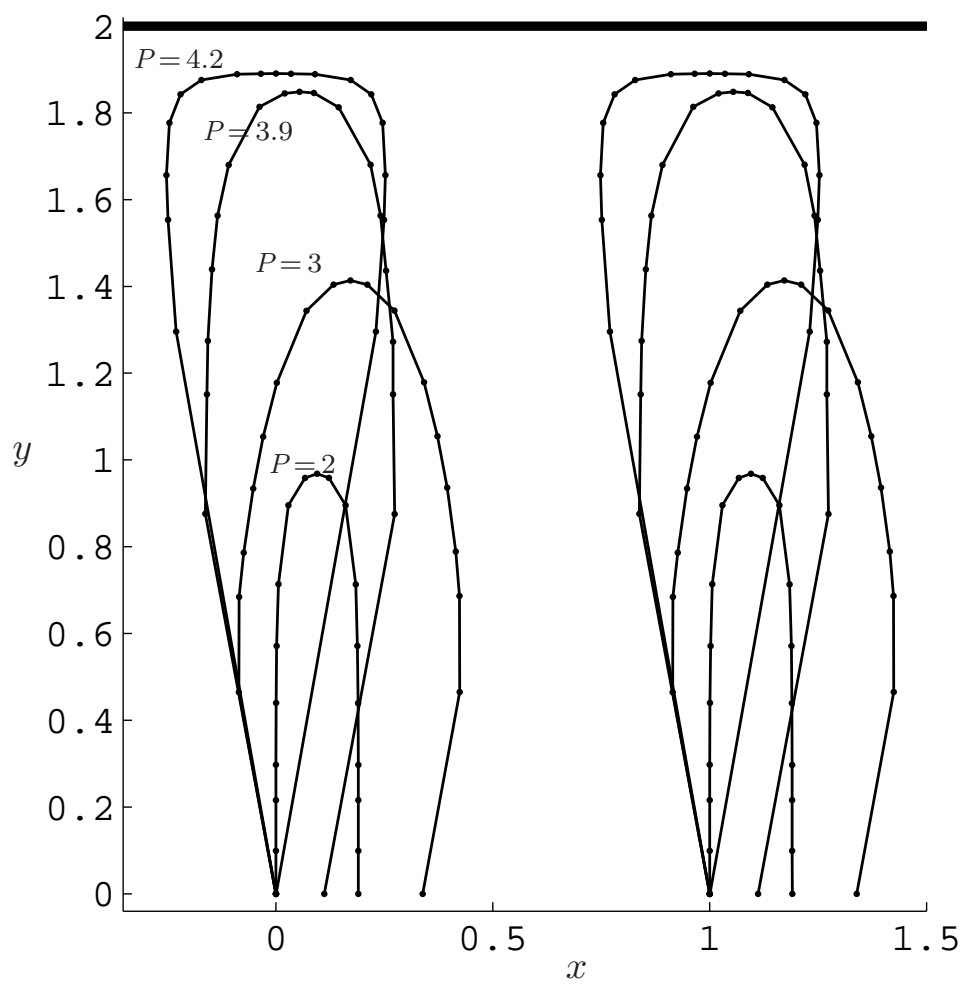

Figure 7: Optimum shapes that maximize the Shape Factor when the dimensionless height is $H=2$ and the $B i=0.1$. The four shapes correspond to $P=2, S=0.89 ; P=3, S=0.0938$; $P=3.9, S=0.098$; and $P=4.2, S=0.099$. 


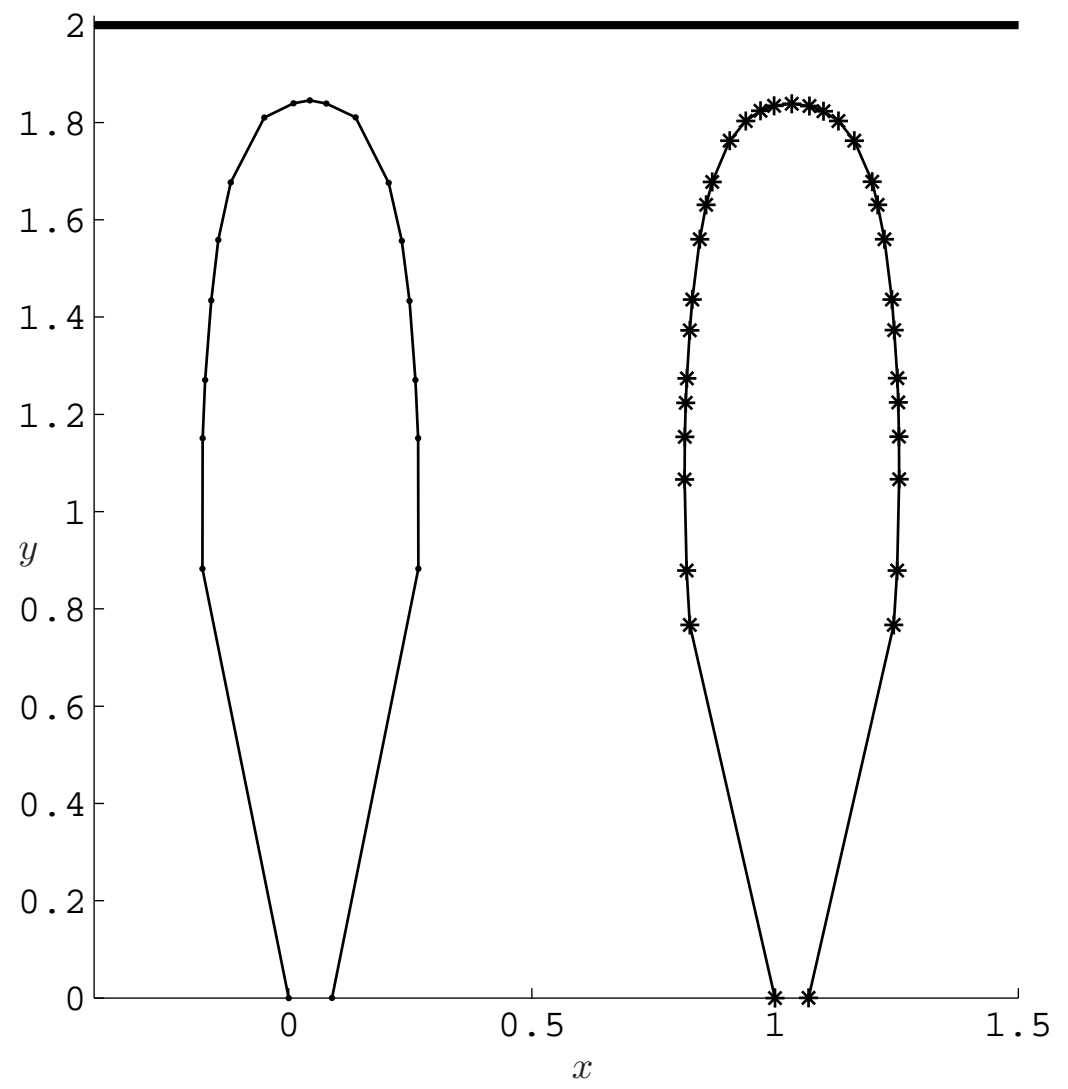

Figure 8: Accuracy of the optimum shape by considering the case $H=2, B i=0.1$ and perimeter $P=3.9$, i.e. the left shape (solid curve with dots) corresponds to one of the shapes of Fig. 7. The right figure (solid curve with stars) corresponds to the same parameters, however we have increased the number of points. In both cases the Shape Factor is $S=0.098$, correct to three decimal places. 


\section{Critical thickness of the slab and critical depth of the pipes}

The existence of a critical thickness, suggested by the Shape Optimization procedure, is further verified through a finite-element numerical simulation [48]. In the finite-element simulation, the domain has been truncated taking advantage of the symmetry of the configuration in the $x$ and $y$-directions (Figs. 4-7). The grid was subdivided until the relative difference of the Shape Factors (Eq. 2) between successive refinements was less than 1\%.

\subsection{Critical thickness}

In Fig. 9, we show a density/contour plot of the temperature field for the case of a periodic array of circular pipes of radius 0.05 embedded in a slab whose upper and lower surfaces are subjected to uniform convection $(B i=0.1)$. The finite element results reveal the existence of a critical thickness which is characterized by maximum Shape Factor, i.e. maximum heat transfer rate (Fig. 10).

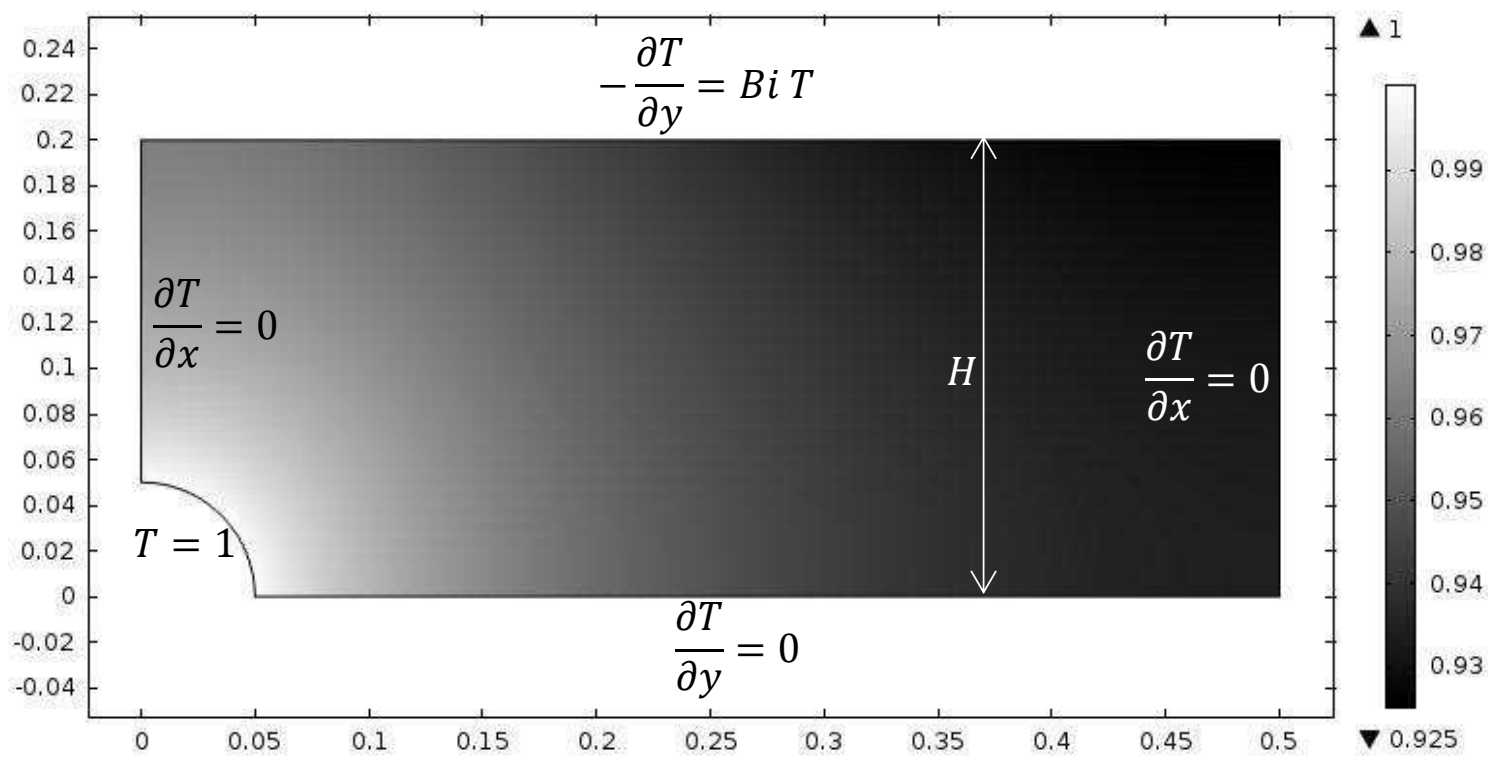

Figure 9: A density/contour plot of the temperature field obtained through finite element numerical simulations [48] $(B i=0.1)$. The domain and the boundary conditions are indicated. Note that, in addition to the symmetry of the domain in the $y$-direction (Fig. 1), we have also taken advantage of the symmetry of the circular pipes in the $x$-direction. 


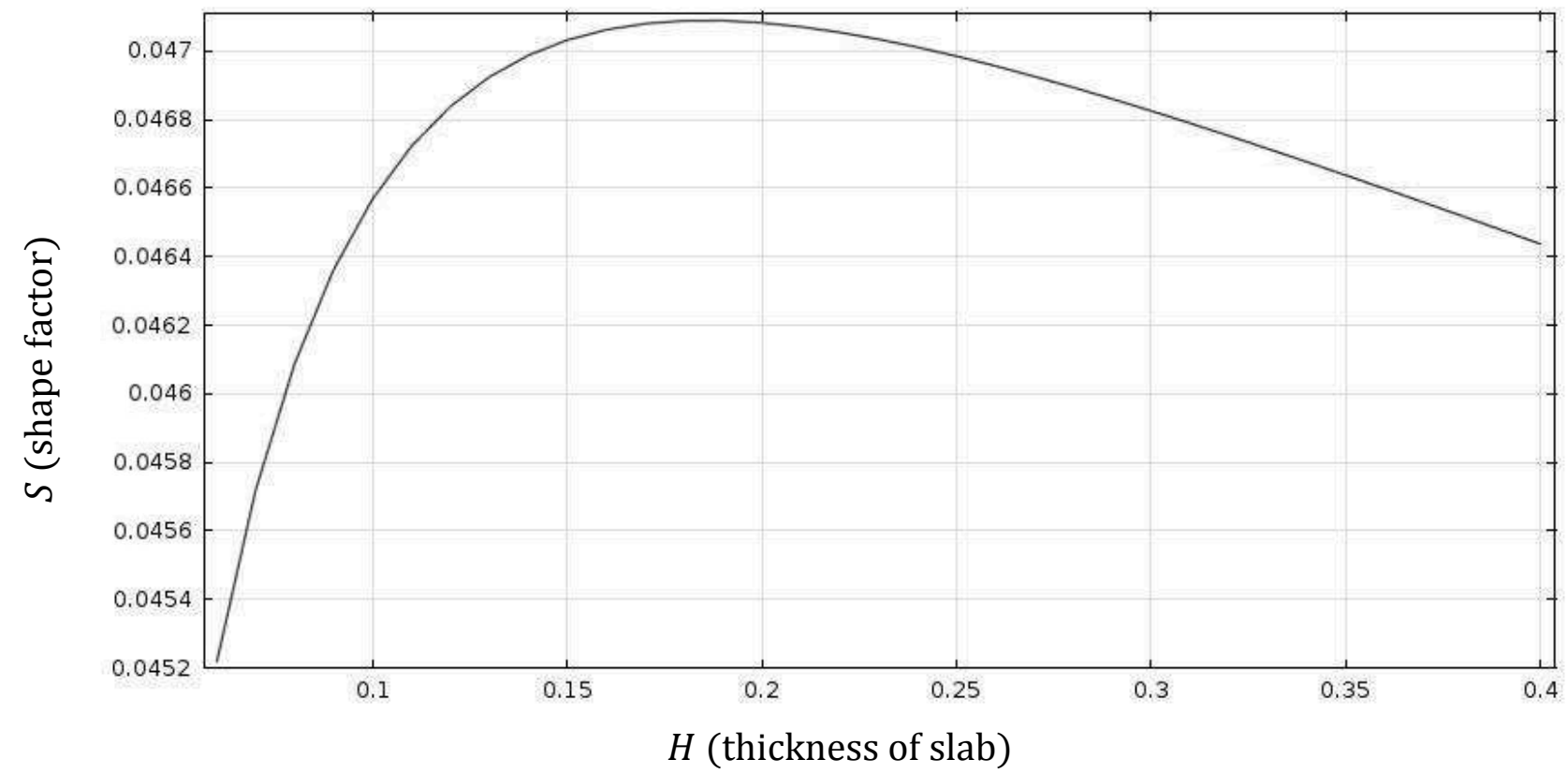

Figure 10: Curve of the Shape Factor $(S)$ versus thickness $(H)$ for a periodic array of circular pipes of dimensionless radius 0.05 , embedded in a slab whose surfaces are subjected to uniform convection $(B i=0.1)$. The critical thickness, where the Shape Factor is maximum, is at $H_{\text {crit }}=0.185$. 


\subsection{Critical depth}

Furthermore, the new family of optimal shapes that do not have their maximum thickness on the center axis (see §3.1), suggests that the optimum shape tends to disconnect from the centerline and approach the upper surface. This is further investigated by considering a circular pipe embedded in an insulated slab (Fig. 11). For comparison, we consider a slab of thickness $H=0.185$, i.e. the critical thickness found in Section $\S 4.1$, embedded with a pipe of radius 0.025, i.e. half the radius considered in Section $§ 4.1$. A plot of the Shape Factor versus the depth $(d)$ of the buried pipe (Fig. 12) suggests that the Shape Factor obtained in Section $\S 3.1$ can be further improved by considering a pipe closer to the surface, as expected. However it is important to note that to achieve maximum heat transfer, the upper part of the pipe should not touch the surface of the slab, rather, the pipe has to be buried below the surface $(d=0.04$, see Fig. 12). 


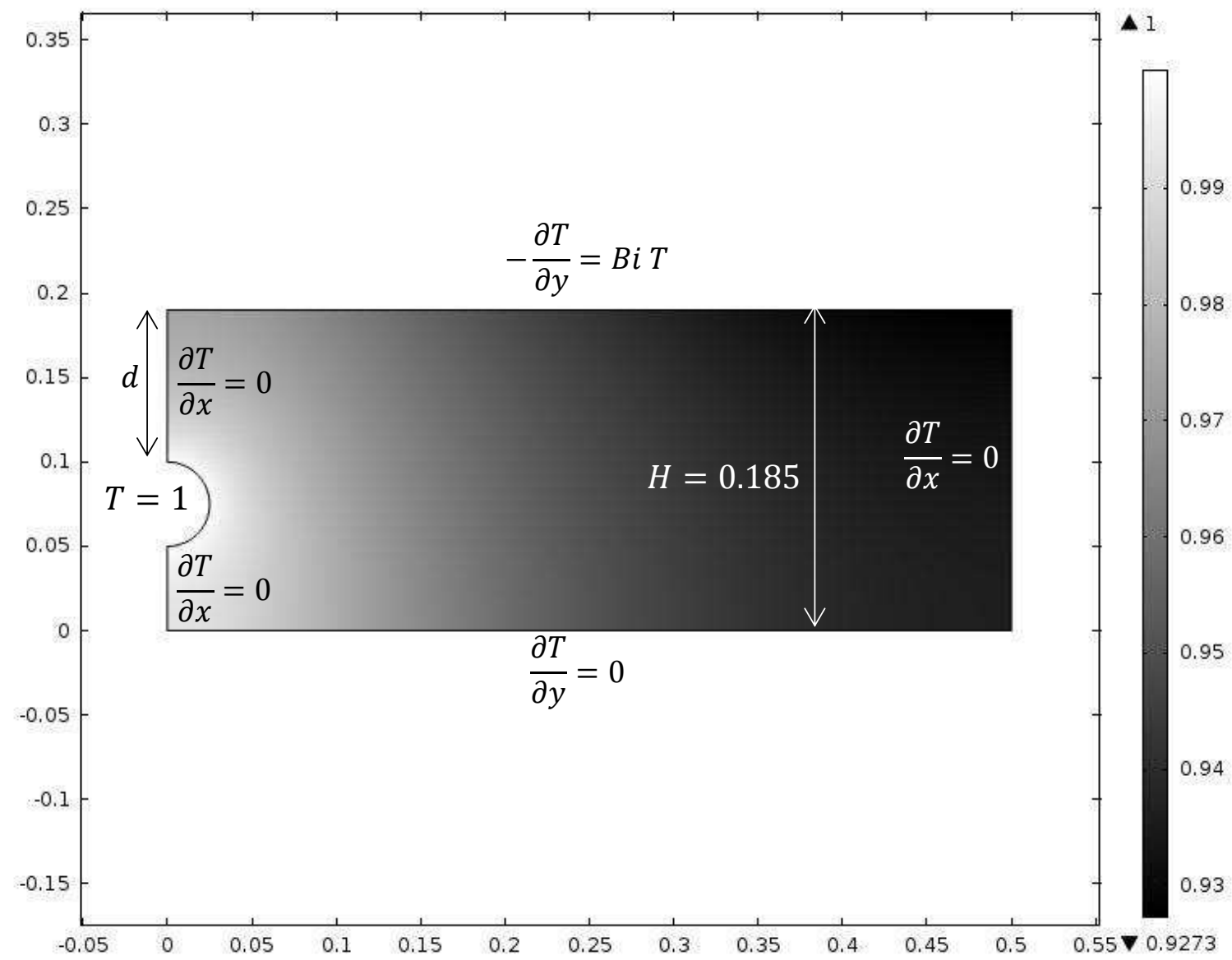

Figure 11: A density/contour plot of the temperature field obtained through finite element numerical simulations [48] $(B i=0.1)$ of a pipe buried in an insulated slab. The domain and the boundary conditions are indicated. Note that we have taken advantage of the symmetry of the circular pipe in the $x$-direction. 


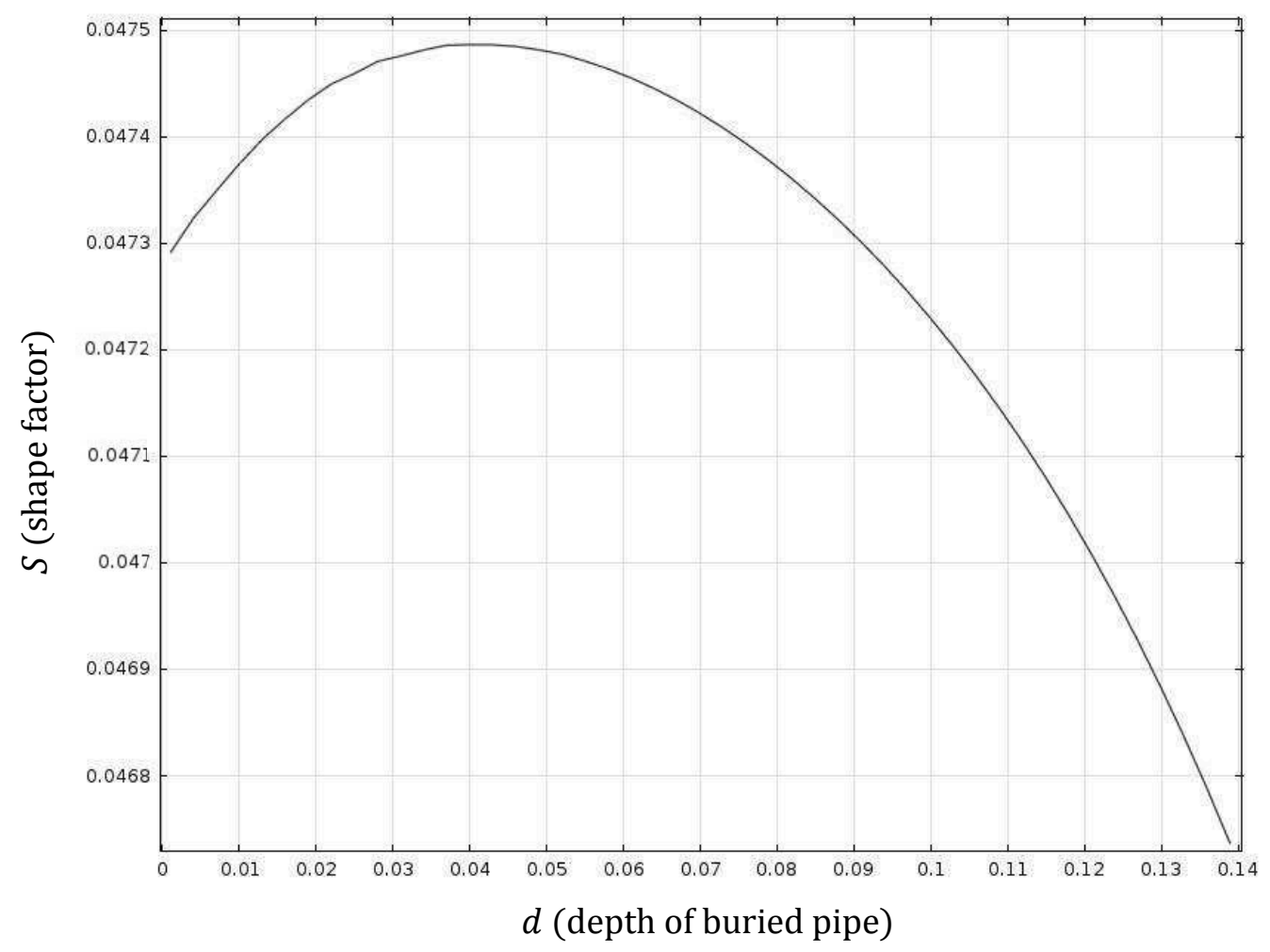

Figure 12: Curve of the Shape Factor $(S)$ versus depth $(d)$ for a periodic array of circular pipes of dimensionless radius 0.025, embedded in an insulate slab whose upper surface is subjected to uniform convection (Fig. 11). The critical depth, where the Shape Factor is maximum, is at $d_{\text {crit }}=0.04$. 


\section{Conclusions}

We have addressed the problem of heat conduction in a solid slab embedded with a periodic array of isothermal, symmetric pipes at the midsection. The upper and lower surfaces of the slab are subjected to uniform convection. The formulation is also relevant for a periodic array of isothermal objects placed along an insulated substrate. The heat conduction problem, which is governed by the Laplace equation, is addressed using conformal mapping techniques and in particular the generalized Schwarz-Christoffel transformation. The transformation maps the domain onto a finite channel, with a variable heat transfer coefficient of uniform distribution, embedded with a periodic array of isothermal strips. The problem in the transformed domain is addressed using the boundary element method to obtain two Fredholm integral equation of the first kind, for the temperature gradient and the temperature along the upper and lower boundaries of the domain. The integral equations are solved numerically to obtain results for the conduction rate and the Shape Factor. The numerical results compare well with asymptotic results.

Subsequently given the thickness of the slab, the Biot number and the perimeter, we pose an isoperimetric Shape optimization problem with the parameters of the generalized Schwarz-Christoffel transformation as the variables of the optimization, and the Shape Factor as the objective function. For large Biot numbers, the optimum shapes agree with the isothermal cases, i.e. circular for sufficiently small perimeters and elliptical elongated towards the surfaces of the slab for larger perimeters. In addition, we were able to discover a new family of optimum shapes for large perimeter and large thickness of the slab; these shapes do not have their maximum width at the mid-section of the slab.

The general conclusion is that the smaller the Biot number, the flatter the optimum shape. Furthermore there exists a critical thickness of the slab and a critical depth of the pipes that is characterized by maximum heat transfer rate.

\section{Acknowledgment.}

The work was funded by Porfyrios Glass Ltd. The authors would like to thank Klaus Schittkowski for providing his NLPQL numerical optimization code [33]. 


\section{A Generalized Schwarz-Christoffel transformation}

Following the analysis in $[17,11]$, we address this problem through a conformal transformation that maps the physical domain onto a simpler domain, where a solution can be more easily obtained. The transformation is realized by discretizing the boundary of a pipe into straight segments and, by applying the generalized Schwarz-Christoffel transformation $[20,22]$, we map the physical domain onto a finite channel (Fig. 2). As described by Davis [20] and Floryan [22] an arbitrary channel, bounded by repeated polygonal segments in the physical domain, can be mapped onto a straight channel in the computational domain using the generalized Schwarz-Christoffel transformation. The transformation is defined uniquely by choosing an arbitrary point $[21,22]$. This is achieved by placing the origin of the transformed domain at the origin of the physical domain $\left(z_{0}=w_{0}=0\right)$. In view of these choices and upon integration the transformation takes the form (Fig. 2)

$$
z[\hat{w} ; \boldsymbol{\alpha}]=R \int_{0}^{\hat{w}} \prod_{l=-\infty}^{l=\infty} \prod_{j=0}^{j=N}\left(\sinh \left[\frac{\pi}{2 \hat{h}}\left(\hat{\theta}-\hat{w}_{j}-l \hat{w}_{N}\right)\right]\right)^{\alpha_{j}} \mathrm{~d} \hat{\theta}
$$

where the inner product identifies the number of elements $(N)$ of the shape, and the infinite outer product the periodic nature of the domain. Similar to $[17,11]$, we normalize the lengths in the complex domain with $w_{N-1}$, which is the length of the pipe in the complex domain. Hence, in the above transformation, $\hat{w}_{j} \mathrm{~s}$ are the normalized images of the $z_{j} \mathrm{~s}$ vertices, $\boldsymbol{\alpha}$ represents the $N+1$-tuple $\alpha_{0}, \alpha_{1}, \alpha_{2}, \ldots, \alpha_{N}$ which are equal to the turning angles multiplied by $\pi$ (the angles are taken to be positive for a clockwise rotation, and $\alpha_{0}$ and $\alpha_{N}$ are defined with respect to the $x$-axis), $R$ is a complex constant, and $\hat{h}=h / w_{N-1}$ is the normalized height of the channel in the transformed domain (without loss of generality we assume that $h=H$ ). For the configurations we will consider $R$ is a real number and can be obtained by requiring that the upper wall of the physical plane, i.e. the line $z=\mathrm{i} H$, transforms to $w=\mathrm{i} h$ :

$$
\operatorname{Im}[z[\mathrm{i} \hat{h} ; \boldsymbol{\alpha}]]=H
$$

In addition, in view of the geometry, we must have

$$
\sum_{j=0}^{N} \alpha_{j}=0 \text { and } \alpha_{N}=0
$$


The parameters $\hat{w}_{j}$ s of the transformation (4), with the exception of $\hat{w}_{0}$ which is equal to zero, do not appear explicitly but given the physical domain, and hence the angles $\pi \alpha_{j}$, a system of non-linear equations must be solved for the unknown parameters [20].

However the optimization problems we will address in Section $§ 3$, similar to the ones formulated in $[16,17,11,18,19]$, are inverse problems. Whereas the classical approach is to compute the $w_{j}$ s, in the Shape Optimization we will need to compute the $\alpha_{j}$ s while the $w_{j}$ s, with the exception of $w_{N-1}$ and $\hat{w}_{N}$, will be defined a priori.

\section{B Boundary element formulation}

We can recast the problem into an integral form by applying Green's theorem $[24,26]$ which for this configuration takes the form:

$$
\int_{0}^{\hat{w}_{N}}\left\{G\left(-\frac{\partial T}{\partial \hat{\eta}^{\prime}}\right)-T\left(-\frac{\partial G}{\partial \hat{\eta}^{\prime}}\right)\right\}_{\hat{\eta}^{\prime}=0} \mathrm{~d} \hat{\xi}^{\prime}+\int_{0}^{\hat{w}_{N}}\left\{G\left(\frac{\partial T}{\partial \hat{\eta}^{\prime}}\right)-T\left(\frac{\partial G}{\partial \hat{\eta}^{\prime}}\right)\right\}_{\hat{\eta}^{\prime}=\hat{h}} \mathrm{~d} \hat{\xi}^{\prime}=0 .
$$

An appropriate Green's function is that associated with a periodic array of sources of period $w_{N}$ located along an insulated surface and a Dirichlet boundary condition along the other surface $[17,11,19]$ :

$$
G\left[\hat{\xi}^{\prime}-\hat{\xi}, \hat{\eta}^{\prime}=0\right]=-\left(\frac{\hat{h}}{\hat{w}_{N}}+\frac{1}{\pi} \sum_{m=1}^{\infty} \frac{1}{m} \tanh \left[2 \pi m \hat{h} / \hat{w}_{N}\right] \cos \left[2 \pi m\left(\hat{\xi}^{\prime}-\hat{\xi}\right) / \hat{w}_{N}\right]\right) .
$$

Unlike [11], where the authors considered isothermal conditions, in this work the conformal mapping transformation has introduced an extra complexity in the upper boundary, i.e. the boundary with the convective boundary condition. To obtain an integral formulation, the Green's theorem is applied twice to obtain two integral equations in terms of both the temperature and the temperature gradient along the upper and lower boundaries; one integral equation is associated with a periodic array of sources located along the lower surface and one with a periodic array of sources located on the upper surface:

$$
\begin{gathered}
\int_{0}^{\hat{w}_{N}} T\left[\hat{\xi}^{\prime}, 0\right] \frac{\partial G}{\partial \hat{\eta}}\left[\hat{\xi}^{\prime}-\hat{\xi}, 0\right] \mathrm{d} \hat{\xi}^{\prime}+B i \int_{0}^{\hat{w}_{N}} G\left[\hat{\xi}^{\prime}-\hat{\xi}, h\right] T\left[\hat{\xi}^{\prime}-\hat{\xi}, \hat{h}\right]\left|\frac{\mathrm{d} z}{\mathrm{~d} \hat{w}}\right|_{\hat{w}=\hat{\xi}^{\prime}+\mathrm{i} \hat{h}} \mathrm{~d} \hat{\xi}^{\prime}-T[\hat{\xi}, \hat{h}]=0 \\
\int_{0}^{\hat{w}_{N}} T\left[\hat{\xi}^{\prime}, \hat{h}\right] \frac{\partial G}{\partial \hat{\eta}}\left[\hat{\xi}^{\prime}-\hat{\xi}, \hat{h}\right] \mathrm{d} \hat{\xi}^{\prime}+\int_{0}^{\hat{w}_{N}} G\left[\hat{\xi}^{\prime}-\hat{\xi}, 0\right] \frac{\partial T}{\partial \hat{\eta}}\left[\hat{\xi}^{\prime}, 0\right] \mathrm{d} \hat{\xi}^{\prime}-T[\hat{\xi}, 0]=0
\end{gathered}
$$

The above two integral equations along with the boundary conditions at $\hat{\eta}=0$ (Eq. 1 ) constitute a consistent set of equations in terms of the variables $T$ and $\partial T / \partial \hat{\eta}$ along the upper and lower boundaries. 
The integral equations are solved numerically using the collocation boundary element method [24]. The boundary integral is desingularized as outlined in [25, 28, 29, 17, 11, 12, $30,19]$, in order to improve the accuracy and the speed of the computational calculation.

\section{References}

[1] F.P. Incropera and D.P. DeWitt, Fundamentals of Heat and Mass Transfer, (John Wiley \& Sons, 1990).

[2] Bau H.H. and Sadhal S.S., Heat losses from a fluid flowing in a buried pipe, Int. J. Heat Mass Transfer 25, 1621 (1982).

[3] Chung M., Jung P-S. and Rangel R.R., Semi-analytical solution for heat transfer from a buried pipe with convection on the exposed surface, Int. J. Heat Mass Transfer 42, 3771 (1999).

[4] Ganzevles F.L.A. and vanderGeld C.W.M., The shapefactor of conduction in a multiple channel slab and the effect of non-uniform temperatures, Int. J. Heat Mass Transfer 40, 2493 (1997).

[5] De Mey G., Temperature distribution in floor heating systems, Int. J. Heat Mass Transfer 23, 1289 (1980).

[6] Ganzevles F.L.A. and vanderGeld C.W.M., On the prediction of condenser plate temperatures in a cross-flow condenser, Exp. Thermal Fluid Science 26, 139 (2002).

[7] J.C. Morud and A. Simonsen, Heat Transfer form partially buried pipes, 16th Australasian Fluid Mechanics Conference, (2007).

[8] Y. Ioannou, M. M. Fyrillas, K. Polychronopoulou and C. Doumanidis, Analytical model for geometrical characteristics control of laser sintered surfaces, Int. J. NanoManufacturing 6, 300 (2010).

[9] F.L.A. Ganzelves and C.W.M. Van Der Geld, The shape factor of conduction in a multiple channel slab and the effect non-uniform temperature, Int. J. Heat Mass Transfer 40, 2493 (1997). 
[10] M. Neophytou, E. Georgiou, M.M. Fyrillas and S.A. Choulis, Two step sintering process and metal grid design optimization for highly efficient ITO free organic photovoltaics, Solar Energy Materials \& Solar Cells 122, 1 (2014).

[11] M.M. Fyrillas, Shape factor and shape optimization for a periodic array of isothermal pipes, Int. J. Heat Mass Transfer 53, 982 (2010).

[12] M.M. Fyrillas and H.A. Stone, Critical insulation thickness of a slab embedded with a periodic array of isothermal strips, Int. J. Heat Mass Transfer 54, 180 (2011).

[13] T. Leontiou and M.M. Fyrillas, Critical thickness of an optimum extended surface characterized by uniform heat transfer coefficient, arXiv:1503.05148 [physics.class-ph] (2015).

[14] M.M. Fyrillas and T. Leontiou, Critical Biot number of a periodic array of rectangular fins, ASME, J. Heat Transfer 138, 024504 (2016).

[15] M. Brady \& C. Pozrikidis, Diffusive transport across irregular and fractal walls, Proc. R. Soc. Lond. A 442, 571 (1993).

[16] M.M. Fyrillas, Shape optimization for 2D diffusive scalar transport, Optimization and Engineering 10, 477 (2009).

[17] M.M. Fyrillas, Heat conduction in a solid slab embedded with a pipe of general crosssection: Shape Factor and Shape Optimization, Int. J. Eng. Sci. 46, 907 (2008).

[18] T. Leontiou, M. Kotsonis and M.M. Fyrillas, Optimum isothermal surfaces that maximize heat transfer, Int. J. Heat Mass Transfer 63, 13 (2013).

[19] T. Leontiou and M.M. Fyrillas, Shape optimization with isoperimetric constraints for isothermal pipes embedded in an insulated slab, ASME, J. Heat Transfer 136, 94502-1-094502-6 (2014).

[20] R.T. Davis, Numerical methods for coordinate generation based on Schwarz-Christoffel transformation, AIAA Paper No. 79-1463, 4th Computational Fluid Dynamics Conference 180 (1979). 
[21] J.M. Floryan, Conformal-mapping-based coordinate generation method for channel flows, Journal of Computational Physics 58, 229 (1985).

[22] J.M. Floryan, Conformal-mapping-based coordinate generation method for flows in periodic configurations, Journal of Computational Physics 62, 221 (1986).

[23] T.A. Driscoll \& L.N. Trefethen, Schwarz-Christoffel Mapping, (Cambridge University Press, 2002).

[24] C. A. Brebbia, Boundary Elements: An Introductory Course (Computational Mechanics; 2nd edition, 1992).

[25] H.A. Stone, Heat/mass transfer from surface films to shear flows at arbitrary Peclet numbers, Phys. Fluids A 1, 1112 (1989).

[26] M.D. Greenberg, Foundations of Applied Mathematics, (Prentice-Hall, 1978).

[27] M.M. Fyrillas and C. Pozrikidis, Conductive heat transport across rough surfaces and interfaces between two conforming media, Int. J. Heat Mass Trans. 44, 1789 (2001).

[28] M.M. Fyrillas, Advection-dispersion mass transport associated with a non-aqueousphase liquid pool, J. of Fluid Mech. 413, 49 (2000).

[29] M.M. Fyrillas and E. J. Kontoghiorghes, Numerical calculation of mass transfer from elliptical pools in uniform flow using the boundary element method, Trans. Porous Media 55, 91 (2004).

[30] Y. Ioannou, M.M. Fyrillas and H. Doumanidis, Approximate Solution to Fredholm Integral Equations using Linear Regression and Applications to Heat and Mass Transfer, newblockEng. Anal. Bound. Elem. 36, 1278 (2012).

[31] E. Hahne and U. Grigull, Shape factor and shape resistance for steady multidimensional heat conduction, Int. J. Heat Mass Transfer, 18, 751 (1975).

[32] R. Fletcher, Practical Methods of Optimization, (John Wiley and Sons, 1987).

[33] K. Schittkowski, NLPQL: A Fortran subroutine for solving constrained nonlinear programming problems, Annals of Operations Research 5, 485 (1985/86). 
[34] K. A. Belibassakis, Th.P. Gerostathis, K.V. Kostas, C.G. Politis, P.D. Kaklis, A.I. Ginnis, and C. Feurer, A BEM-ISOGEOMETRIC method for the ship wave-resistance problem, J. of Ocean Engineering 60, 53-67 (2013).

[35] A.-A.I. Ginnis, K.V. Kostas, C.G. Politis, P.D. Kaklis, K.A. Belibassakis, Th.P. Gerostathis, M.A. Scott and T.J.R Hughes, Isogeometric Boundary-Element Analysis for the Wave-Resistance Problem using T-splines, Journal of Computer Methods in Applied Mechanics and Engineering 279, 425-439 (2014).

[36] K.V. Kostas, A.I. Ginnis, C.G. Politis, and P.D. Kaklis, Ship-Hull Shape Optimization with a T-Spline based BEM-Isogeometric Solver, Journal of Computer Methods in Applied Mechanics and Engineering, Isogeometric Analysis Special Issue, 284, 611-622 (2015), (http://dx.doi.org/10.1016/j.cma.2014.10.030).

[37] C.G. Politis, A. Papagiannopoulos, K.A. Belibassakis, P.D. Kaklis, K.V. Kostas, A.I. Ginnis and T.P. Gerostathis, An isogeometric BEM for exterior potential-flow problems around lifting bodies, 11th World Congress on Computational Mechanics (WCCM XI), 20-25 July 2014, Barcelona, Spain.

[38] Y. Parte, D. Auroux, J. Clement, M. Masoudi and J. Hermetz, Collaborative Optimization, Design Optimization in Computational Mechanics, Wiley-ISTE, 321 (2010).

[39] J.A-C. G. Iaccarino, G. Petrone and V. Sellapan, Extreme ensemble computations for optimization under uncertainty, Evolutionary and deterministic methods for design, optimization and control, C. Poloni, D. QUagliarella, J. Périaux, N. Gauger and K. Yiannakoglou (Eds.), CIRA, Capua, Italy (2011).

[40] L. Wei, S.X.J. Shao, C.H. Dai and H.R. He, Coupling mesh morphing and parametric shape optimization using simuopti, 3rd ANSA \& ETA International Conference, (2009).

[41] J.T. Allison, M. Kokkolaras, M. Zawislak and P. Papalambros, On the use of analytical target cascading and collaborative optimization for complex system design, 6th World congress on Structural and Multidisciplinary Optimization, Rio de Janeiro, 30 May-03 June (2005). 
[42] O. Pironneau, Optimal Shape Design for Elliptic Systems, (Springer, New York, 1984).

[43] J. Haslinger and R.A.E. Mokinen, Introduction to Shape Optimization: Theory, Approximation, and Computation, (SIAM, 2003).

[44] B. Mohammadi and O. Pironneau Applied Shape Optimization for Fluids, Oxford University Press, 2001.

[45] P.M. Morse and H. Feshbach, Methods of Theoretical Physics, Part II, McGraw-Hill Science/Engineering/Math, 1953.

[46] A. Kacimov, Optimal shape of a variable condenser, Proc. R. Soc. Lond. A, 457, 485 (2001).

[47] P. Ya Polubarinova-Kochina, Theory of ground-water movement, (Princeton University Press 1962).

[48] COMSOL, Heat Transfer Module, COMSOL Multiphysics User's Guide, 2008. Version 3.4, COMSOL AB, Stockholm, Sweden. 